\title{
Syncope and an Intra-Hisian Atrioventricular Block After Radiation Therapy: Electrophysiologic Observations and Review of Literature
}

\author{
Theresa Ratajczak $^{\mathrm{a}, \mathrm{b}}$, Umbreen Hussain ${ }^{\mathrm{a}}$, Abdul Wase ${ }^{\mathrm{a}}$
}

\begin{abstract}
We present a case of a patient with recurrent syncope of sudden onset. She had a positive passive head-up tilt test for a mixed type of neurocardiogenic syncope. Additionally, she developed a narrow complex tachycardia on telemetry monitoring. Electrophysiology study revealed an intra-Hisian delay which upon provocation with programmed electrical stimulation, developed into a complete intraHisian atrioventricular block. Based on past medical history of radiation therapy for adenocarcinoma of the breast, the etiology of heart block was attributed to the remote effect of radiation-induced cardiac toxicity. Positive head-up tilt test for neurocardiogenic syncope and supraventricular tachycardia were incidental findings that could have confounding influence on management of such patients.
\end{abstract}

Keywords: Intra-Hisian block; Atrioventricular block; Complete heart block; Radiation therapy

\section{Introduction}

The presence of a narrow QRS complex on an electrocardiogram (EKG) draws less attention to the consideration of conduction system disease in patients presenting with syncope. However, in patients with prior history of radiation therapy to the chest wall, it is particularly important to carefully evaluate for progressive fibrosis of the conduction system, which may present several years after the completion of treatment.

\section{Case Report}

A 53-year-old woman presented to the hospital with four epi-

\section{Manuscript submitted December 12, 2018, accepted April 8, 2019}

a Department of Internal Medicine, Wright State University Boonshoft School of Medicine, Dayton, OH, USA

${ }^{b}$ Corresponding Author: Theresa Ratajczak, Department of Internal Medicine, Wright State University Boonshoft School of Medicine, Dayton, OH, USA. Email: theresa.m.ratajczak@gmail.com

doi: https://doi.org/10.14740/jmc3233 sodes of syncope of sudden onset occurring over a 3-week period. During one of these episodes, she fell backward and sustained a blunt injury to the back of her head. Her past medical history was significant for adenocarcinoma of the left breast diagnosed 11 years prior which was treated with mastectomy and radiation therapy. Patient's review of symptoms, physical examination and laboratory results were unremarkable. She denied any prodromal or postictal symptoms. Computed tomography (CT) scan of the head, electroencephalogram, echocardiogram and carotid Doppler studies were normal. A 12-lead EKG demonstrated sinus rhythm with a narrow QRS complex (Fig. 1a).

The patient was admitted to the hospital for observation and further evaluation and workup. Review of the overnight telemetry demonstrated the development of a first-degree atrioventricular block (Fig. 1b) as well as a narrow complex tachycardia with RP interval of $140 \mathrm{~ms}$ (Fig. 1c). She was referred for tilt table testing followed by an electrophysiologic evaluation. A passive head-up tilt test was performed with an arterial line inserted for blood pressure monitoring. During the baseline supine state, patient's blood pressure was $163 / 70 \mathrm{~mm}$ $\mathrm{Hg}$ with a corresponding heart rate of 106 beats/min. She was then tilted up to $70^{\circ}$ and developed a mixed type of neurocardiogenic syncope after $4 \mathrm{~min}$. Her blood pressure fell to 71/26 $\mathrm{mm} \mathrm{Hg}$ with the heart rate dropping to 20 beats $/ \mathrm{min}$. These events were associated with recurrence of her symptoms, and she experienced a syncopal episode.

An electrophysiology (EP) study was carried out using the standard protocol. Three quadripolar pacing electrode catheters were introduced via the left femoral venous approach and were placed in the high right atrium, atrioventricular junction across the tricuspid valve and right ventricular apex. A decapolar deflectable coronary sinus catheter was introduced via the right femoral approach and advanced under fluoroscopic and electrocardiographic guidance into the coronary sinus. Pacing was performed. The patient's sinus cycle length was $710 \mathrm{~ms}$, AH interval was $120 \mathrm{~ms}$ and HV interval was $110 \mathrm{~ms}$. On decremental atrial pacing from the high right atrium, an atrioventricular Wenckebach block occurred at $400 \mathrm{~ms}$. No VA conduction was noted by pacing from the right ventricular apex at a drive cycle length of 650 $\mathrm{ms}$. Intra-Hisian delay of $65 \mathrm{~ms}$ was noted during the baseline recording (Fig. 2a).

On programmed electrical stimulation from the high right atrium, the patient developed a complete intra-Hisian block 


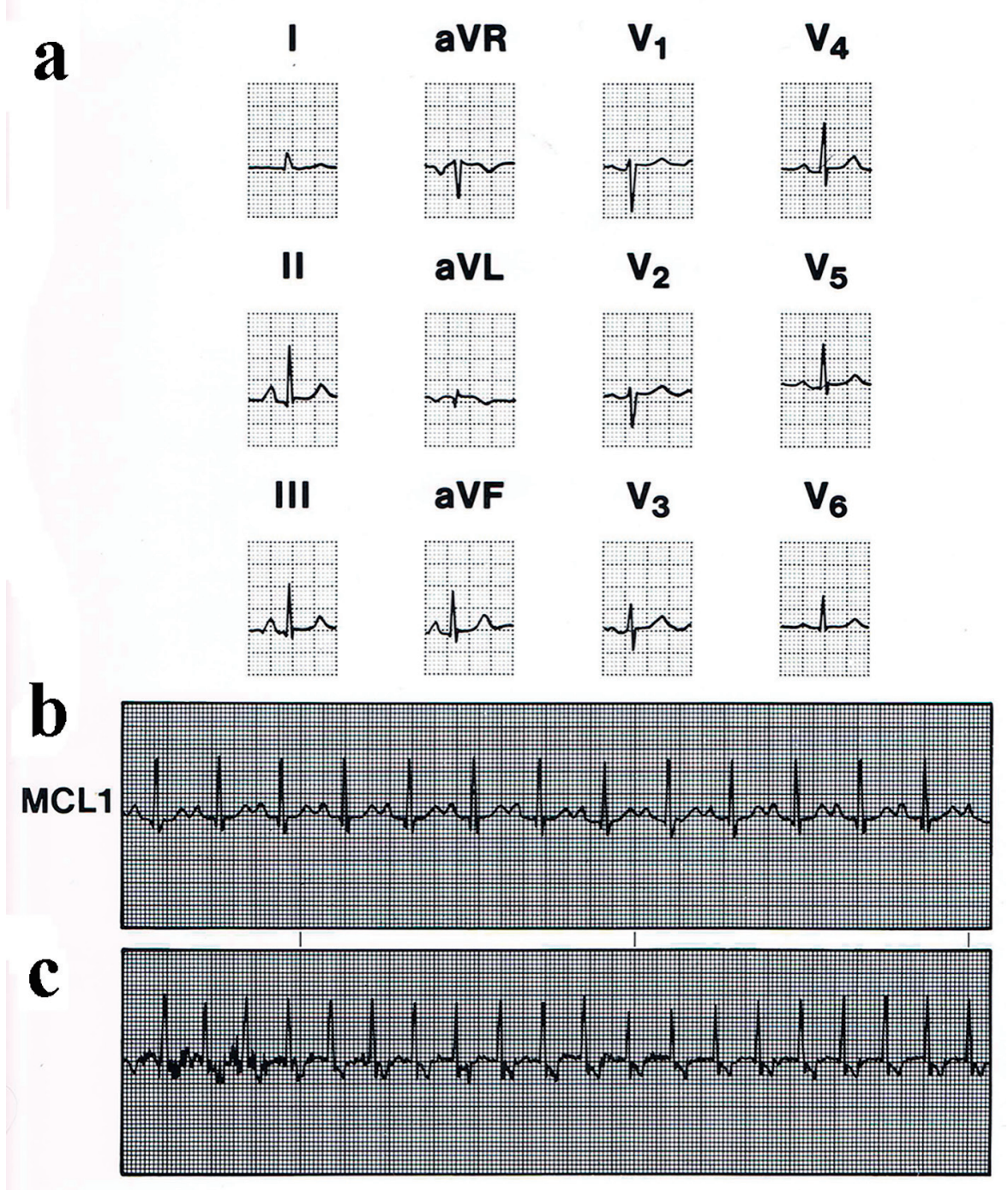

Figure 1. (a) Patient's presenting 12-lead electrocardiogram demonstrated sinus rhythm with a narrow QRS complex. (b) Telemetry demonstrating first-degree atrioventricular block. (c) Telemetry with narrow complex tachycardia.

(Fig. 2b). Supraventricular tachycardia could not be induced, despite starting an isoproterenol infusion at $10 \mu \mathrm{g} / \mathrm{min}$. After a washout period of $10 \mathrm{~min}$, procainamide infusion was initiated. A spontaneous complete intra-Hisian atrioventricular block (Fig. 3) developed after $350 \mathrm{mg}$ of procainamide was infused. Temporary ventricular pacing was started. The patient subsequently underwent a permanent dual chamber pacemaker implantation.

The differential diagnosis for the development of the intra-Hisian atrioventricular block in our patient included degeneration of the conduction system, myocardial infarction, proliferative obstruction of the coronary artery supplying the atrioventricular node, congenital etiology, surgery or trauma. These were all considered as possibilities; however, due to her history of mediastinal radiation for breast malignancy, this was thought to be the most plausible underlying etiology. She was discharged home in a stable condition with a close cardiology follow-up. She was noted to be asymptomatic with resolution of syncope on a follow-up visit. The pacemaker telemetry showed $43 \%$ dependence on ventricular pacing; no arrhythmias were noted.

\section{Discussion}

Disease in the bundle of His (an intra-His block) is an uncommon entity that presents a diagnostic challenge to the clinician. The presence of a narrow QRS complex on an EKG draws less attention to the consideration of conduction system disease in patients presenting with syncope. This is unfortunate, as patients with an intra-Hisian block are at high risk for risk of syncope [1]. The formal diagnosis is usually made with an EP study.

Radiation therapy is known to cause significant abnormalities of the pericardium, myocardium, valves and the conduction system [2]. Radiation therapy causes intense fibrosis in 



Figure 2. (a) Baseline recording in electrophysiology study. (b) Programmed electrical stimulation from high right atrium showing development of a complete intra-Hisian block.

the cardiac conduction system, which is also noted elsewhere such as in the pericardium and the myocardium [3, 4]. Autopsy reports cite severe fibrosis of the conduction system present in patients following radiation therapy [4]. This destroys the typical architecture of the conduction system, leading to varying degrees of atrioventricular block. Patients generally develop left bundle branch block (LBBB), right bundle branch block or a trifascicular block before developing a complete atrioventricular block. Conduction abnormalities usually present several years after radiation therapy has been completed. A delay of about 10 - 20 years after completion of treatment is reported [2]. Varying degrees of atrioventricular blocks due to prior radiation therapy to the mediastinum have been described in the literature [2-4].

To our knowledge, our report is the first known case of an intra-Hisian block due to prior radiation treatment. Patients with an intra-Hisian block typically have a prolonged PR interval reflecting the delay in conduction of both the atrioventricular node and the His bundle, sparing the His-Purkinje system. Because of this, a narrow QRS results as was the case in our patient.

In a recent retrospective study of patients with paroxysmal atrioventricular blocks with intra-Hisian delay/blocks, it was concluded that these patients rarely progress to pacemaker dependence during long-term follow-up [1]. Our patient however required a high percentage of ventricular pacing $(43 \%)$. Perhaps, because of prior radiation therapy, there was more extensive damage to the atrioventricular conduction system as evident by the presence of additional first-degree atrioventricular block.

Initially, our index of suspicion for an advanced atrioventricular block was low due to the narrow QRS complexes on the surface EKG. However, the occurrence of a first-degree atrioventricular block and supraventricular tachycardia prompted us to proceed with an EP study.

We could have missed the diagnosis completely, had we not pursued the study and relied solely on the findings of the tilt table testing. However, in the setting of surface EKG changes further cardiovascular workup for etiology of syncope is considered to be the standard of care. While we cannot de-

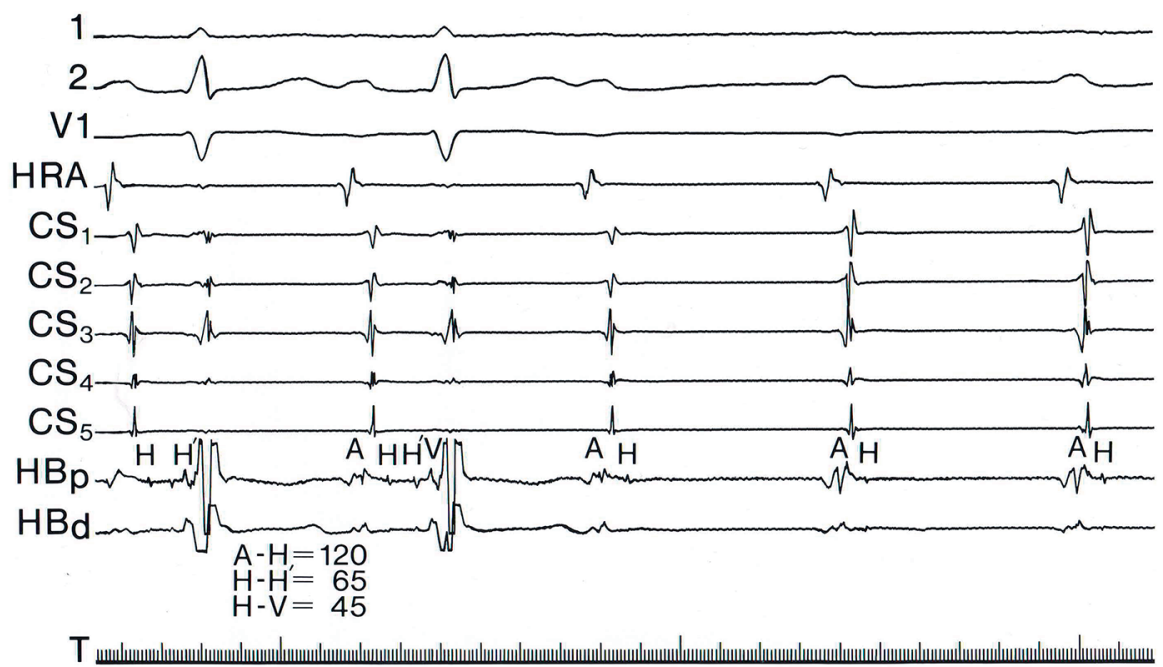

Figure 3. Spontaneous complete intra-Hisian atrioventricular block after procainamide infusion. 
finitively establish the mechanism of syncope to be strictly due to the intra-Hisian block, its abrupt onset and history of remote radiation therapy are a supportive evidence for consideration of this to be the most likely etiology.

Review of literature in patients who received radiation therapy shows that the following risks factors can help to identify those who may potentially develop heart block: 1) Total radiation dose received to the heart of more than $40 \mathrm{~Gy}$; 2) Delay of 10 years or more since completion of therapy; 3 ) Conduction abnormalities on surface EKG (e.g. varying degree of atrioventricular block, LBBB); 4) Prior pericardial involvement; 5) Associated cardiac or mediastinal lesions [5].

\section{Conclusion}

Our patient most likely developed syncope following an intraHisian atrioventricular block due to remote radiation therapy for adenocarcinoma of the breast. Her presentation was confounded by a positive tilt table test as well as the presence of a first-degree atrioventricular block and a narrow complex tachycardia. Frequently more than one mechanism of syncope may coexist and may have confounding influence on the management of such patients. It is essential to keep a broad differential diagnosis and perform a thorough history and physical examination in patients presenting with syncope, particularly if they have a history of radiation to the chest wall.

\section{Key teaching points}

1) The presence of narrow QRS complexes draws less attention to consideration of conduction system disease in patients presenting with syncope. However, an intra-Hisian block should be considered in the differential in patient with certain risk factors as they are at high risk for syncope.

2) Patients with history of prior radiation to the thorax especially those who received more than 40 Gy of radiation, conduction abnormalities on surface EKG, pericardial involvement, cardiac or mediastinal lesions and who are more than 10 years from completion of therapy are at a higher risk for developing a heart block.

3) Conduction abnormalities usually present several years after radiation therapy has been completed. A delay of about 10 - 20 years after completion of therapy is reported.

\section{Acknowledgments}

We would like to acknowledge Wright State University and
Good Samaritan Hospital in Dayton, Ohio for their support in this manuscript.

\section{Financial Disclosure}

None to declare.

\section{Conflict of Interest}

All authors have no conflict of interest to report.

\section{Informed Consent}

Informed consent was obtained from the patient for publication of this case report. The patient was informed that this information will be published without any personal identifiers in the text and images published.

\section{Author Contributions}

All authors contributed in the case writing, data gathering and literature review for this case report.

\section{References}

1. Ragupathi L, Johnson D, Greenspon A, Frisch D, Ho RT, Pavri BB. Clinical and electrophysiological characteristics of patients with paroxysmal intra-His block with narrow QRS complexes. Heart Rhythm. 2018;15(9):13721377.

2. Benoff LJ, Schweitzer P. Radiation therapy-induced cardiac injury. Am Heart J. 1995;129(6):1193-1196.

3. Cohen SI, Bharati S, Glass J, Lev M. Radiotherapy as a cause of complete atrioventricular block in Hodgkin's disease. An electrophysiological-pathological correlation. Arch Intern Med. 1981;141(5):676-679.

4. Kaplan BM, Miller AJ, Bharati S, Lev M, Martin Grais I. Complete AV block following mediastinal radiation therapy: electrocardiographic and pathologic correlation and review of the world literature. J Interv Card Electrophysiol. 1997;1(3):175-188.

5. Slama MS, Le Guludec D, Sebag C, Leenhardt AR, Davy JM, Pellerin DE, Drieu LH, et al. Complete atrioventricular block following mediastinal irradiation: a report of six cases. Pacing Clin Electrophysiol. 1991;14(7):1112-1118. 